\title{
A distributed finite-time observer for linear systems
}

\author{
Haik Silm, Rosane Ushirobira, Denis Efimov, Jean-Pierre Richard, Wim Michiels
}

\begin{abstract}
In this paper, the problem of distributed estimation for a linear large-scale system is studied. A nonlinear distributed observer is proposed, whose estimation error converges to zero in a finite time. A fixed-time converging version of the observer is also presented. The efficiency of estimators is demonstrated by computer simulations.
\end{abstract}

\section{INTRODUCTION}

The problem of state estimation for dynamical systems has a long history of research and many extensions were obtained for various models: linear, nonlinear, switched, sampled, time-delay, etc., [1]-[4]. The majority of the proposed observers in all solutions aim to optimize essential properties in their design, such as robustness to measurement noises, overshooting of estimates, time of convergence, tolerance to model uncertainties and parametric errors [5], [6]. Conventional solutions, starting from seminal observers of Luenberger and Kalman, have asymptotic rates of convergence (e.g. the estimates converge to their ideal values with time approaching infinity in the noise-free case). In recent years, many works [7]-[11] deal with finite-time or fixedtime converging observers, meaning that the estimation error becomes zero in a given finite time. In most cases, such estimators are highly nonlinear, and their stability analysis is rather complicated.

The Fourth Industrial Revolution and Internet of Things bring us networked distributed systems (ubiquitous in power production, transportation networks, industrial control processes, for example), when small sensors and actuators are installed everywhere. Using available capacities and simple embedded software, they attempt to enlarge their functionality using possible connections with neighbors and by exchanging available information. For the state estimation this gives rise to the problem of a distributed observer design [12]-[17], where the same (probably large-scale) system is estimated by a set of local observers having different local measured information, and which are able to exchange the calculated estimates in the network to recuperate the full system state. In other words, the idea of distributed estimation is originated by the fact that from each particular sensor

The first four authors are with Inria, Non-A team, Parc Scientifique de la Haute Borne, 40 avenue Halley, 59650 Villeneuve d'Ascq, France and CRIStAL (CNRS UMR 9189), Centrale Lille, Cité Scientifique, 59651 Villeneuve d'Ascq, France. W. Michiels and H. Silm are with the Department of Computer Science, KU Leuven, Celestijnenlaan 200A, 3001 Heverlee, Belgium.

This work has been partially supported by the project $\mathrm{UCoCoS}$, funded by the European Union's Horizon 2020 research and innovation programme under the Marie Skłodowska-Curie Grant Agreement No 675080, by Russian Science Foundation (Project 17-19-01422), the Government of Russian Federation (Grant 074-U01) and the Ministry of Education and Science of Russian Federation (Project 14.Z50.31.0031). the system state may be unobservable, but using coupling a distributed complete state observer can be designed.

To the best of our knowledge, the proposed distributed observers in the literature are linear with linear output injections and coupling laws, leading to asymptotic convergence rates of the estimation error. The goal of this work is to propose a nonlinear distributed observer converging in a finite/fixed-time to the ideal estimates (in the noise-free case). The proposed solution is an extension of [9], [18] to the distributed scenario. The paper presents qualitative results on finite-time convergence. Future researches include the quantitative analysis and tuning rules.

The paper outline is as follows. Some preliminary results and notions on stability properties and homogeneity are introduced in Section II. The main steps of [9] are reviewed in Section III, with a mild improvement by stating an explicit bound for the degree of homogeneity. The distributed finitetime converging observer design and analysis are given in Section IV. The results of numeric experiments are presented in Section V. Concluding remarks and future works are discussed in Section VI.

\section{Notation}

- The operation $\lceil x\rfloor^{\alpha}$ with $x, \alpha \in \mathbb{R}^{n}, \alpha>0$ denotes the sign preserving element-wise exponentiation, i.e. $\lceil x\rfloor^{\alpha}=\left[\begin{array}{lll}\operatorname{sign}\left(x_{1}\right)\left|x_{1}\right|^{\alpha_{1}} & \ldots & \operatorname{sign}\left(x_{n}\right)\left|x_{n}\right|^{\alpha_{n}}\end{array}\right]^{\mathrm{T}}$.

- $1_{n} \in \mathbb{R}^{n}$ is the vector with all entries equal $1, I_{n}$ is the $n \times n$ identity matrix.

- For $x=\left[\begin{array}{lll}x_{1} & \ldots & x_{n}\end{array}\right]^{\mathrm{T}} \in \mathbb{R}^{n}, \operatorname{diag}(x)$ denotes the diagonal matrix with $x_{1}, \ldots, x_{n}$ on the main diagonal and 0 otherwise, and $\operatorname{diag}\left\{B_{i}\right\}_{i=1}^{N}$ is a $N p \times N q$ block diagonal matrix with blocks $B_{1}, \ldots, B_{N} \in \mathbb{R}^{p \times q}$ on the main diagonal.

- $\lambda_{\min }(P)$ and $\lambda_{\max }(P)$ are the smallest and the greatest eigenvalues of a real symmetric matrix $P$, respectively.

- A sequence of integers $1,2, \ldots, n$ is denoted by $\overline{1, n}$.

- For a closed set $S \in \mathbb{R}^{n}$, denote its border as $\partial S$.

- A continuous function $\alpha: \mathbb{R}_{+} \mapsto \mathbb{R}_{+}$belongs to the class $\mathcal{K}$ if $\alpha(0)=0$ and the function is strictly increasing, and belongs to the class $\mathcal{K}_{\infty}$ if $\alpha \in \mathcal{K}$ and it is increasing to infinity.

\section{PRELIMINARIES}

Consider a generic nonlinear system:

$$
\dot{x}(t)=f(x(t)), t \geq 0,
$$

where $x(t) \in \mathbb{R}^{n}$ is the state, $f: \mathbb{R}^{n} \rightarrow \mathbb{R}^{n}$ ensures forward existence and uniqueness of the system solutions (at least locally) and $f(0)=0$. For an initial condition $x_{0} \in \mathbb{R}^{n}$, 
denote the corresponding solution by $X\left(t, x_{0}\right)$ for any $t \geq 0$ for which the solution exists.

Following [3], [19], [20], let $\Omega$ be an open neighborhood of the origin in $\mathbb{R}^{n}$.

Definition 1: At the steady state $x=0$ the system (1) is said to be

(a) stable if for any $x_{0} \in \Omega$ the solution $X\left(t, x_{0}\right)$ is defined for all $t \geq 0$, and for any $\epsilon>0$ there is $\delta>0$ such that for any $x_{0} \in \Omega$, if $\left\|x_{0}\right\| \leq \delta$ then $\left\|X\left(t, x_{0}\right)\right\| \leq \epsilon$ for all $t \geq 0$;

(b) asymptotically stable if it is stable and for any $\kappa>0$ and $\epsilon>0$ there exists $T(\kappa, \epsilon) \geq 0$ such that for any $x_{0} \in \Omega$, if $\left\|x_{0}\right\| \leq \kappa$ then $\left\|X\left(t, x_{0}\right)\right\| \leq \epsilon$ for all $t \geq T(\kappa, \epsilon)$

(c) finite-time stable if it is stable and finite-time converging from $\Omega$, i.e. for any $x_{0} \in \Omega$ there exists $0 \leq T<+\infty$ such that $X\left(t, x_{0}\right)=0$ for all $t \geq T$. The function $T_{0}\left(x_{0}\right)=\inf \left\{T \geq 0: X\left(t, x_{0}\right)=0 \forall t \geq T\right\}$ is called the settling time of the system (1);

(d) fixed-time stable if it is finite-time stable and $\sup _{x_{0} \in \Omega} T_{0}\left(x_{0}\right)<+\infty$.

The set $\Omega$ is called the domain of stability/attraction.

If $\Omega=\mathbb{R}^{n}$, then the corresponding properties are called global stability/asymptotic stability/finite-time/fixedtime stability of (1) at $x=0$.

Similarly, these stability notions can be defined with respect to a compact invariant set, by replacing the distance to the origin in Definition 1 with the distance to the invariant set.

\section{A. Weighted homogeneity}

Following [21], [22], for strictly positive numbers $r_{i}, i=$ $\overline{1, n}$ called weights and $\lambda>0$, define:

- the vector of weights $\mathbf{r}=\left[\begin{array}{lll}r_{1} & \ldots & r_{n}\end{array}\right]^{T}, r_{\max }=$ $\max _{1 \leq j \leq n} r_{j}$ and $r_{\min }=\min _{1 \leq j \leq n} r_{j}$;

- the dilation matrix function $\Lambda_{r}(\bar{\lambda})=\operatorname{diag}\left\{\lambda^{r_{i}}\right\}_{i=1}^{n}$. Note that $\forall x \in \mathbb{R}^{n}$ and $\forall \lambda>0$ we have $\Lambda_{r}(\lambda) x=$ $\left[\begin{array}{lll}\lambda^{r_{1}} x_{1} & \ldots & \lambda^{r_{n}} x_{n}\end{array}\right]^{T}$;

- the $\mathbf{r}$-homogeneous norm $\|x\|_{r}=\left(\sum_{i=1}^{n}\left|x_{i}\right|^{\frac{\rho}{r_{i}}}\right)^{\frac{1}{\rho}}$ for any $x \in \mathbb{R}^{n}$ and $\rho \geq r_{\max }$. This is not a norm in the standard sense, since the triangle inequality is not satisfied for $\|\cdot\|_{r}$, however there exist $\underline{\sigma}, \bar{\sigma} \in \mathcal{K}_{\infty}$ such that

$$
\underline{\sigma}\left(\|x\|_{r}\right) \leq\|x\| \leq \bar{\sigma}\left(\|x\|_{r}\right) \quad \forall x \in \mathbb{R}^{n} ;
$$

- the sphere and the ball in the homogeneous norm $S_{r}(\rho)=\left\{x \in \mathbb{R}^{n}:\|x\|_{r}=\rho\right\}$ and $B_{r}(\rho)=\{x \in$ $\left.\mathbb{R}^{n}:\|x\|_{r} \leq \rho\right\}$ for $\rho \geq 0$.

Definition 2: A function $g: \mathbb{R}^{n} \rightarrow \mathbb{R}$ is $\mathbf{r}$-homogeneous with degree $\mu \in \mathbb{R}$ if $\forall x \in \mathbb{R}^{n}$ and $\forall \lambda>0$ we have:

$$
\lambda^{-\mu} g\left(\Lambda_{r}(\lambda) x\right)=g(x) .
$$

A vector field $f: \mathbb{R}^{n} \rightarrow \mathbb{R}^{n}$ is $\mathbf{r}$-homogeneous with degree $\nu \in \mathbb{R}$, with $\nu \geq-r_{\min }$ if $\forall x \in \mathbb{R}^{n}$ and $\forall \lambda>0$ we have:

$$
\lambda^{-\nu} \Lambda_{r}^{-1}(\lambda) f\left(\Lambda_{r}(\lambda) x\right)=f(x)
$$

which is equivalent for the $i$-th component of $f$ to be a $\mathbf{r}$-homogeneous function of degree $r_{i}+\nu$.

The system (1) is $\mathbf{r}$-homogeneous of degree $\nu$ if the vector field $f$ is $\mathbf{r}$-homogeneous of the degree $\nu$.

Theorem 1 ([21], [23], [24]): For the system (1) with $\mathbf{r}$-homogeneous and continuous function $f$ the following properties are equivalent:

- the system (1) is (locally) asymptotically stable;

- there exists a continuously differentiable $\mathbf{r}-$ homogeneous Lyapunov function $V: \mathbb{R}^{n} \rightarrow \mathbb{R}_{+}$such that

$$
\begin{gathered}
\alpha_{1}(\|x\|) \leq V(x) \leq \alpha_{2}(\|x\|), \\
L_{f} V(x)=\frac{\partial V}{\partial x}(x) f(x) \leq-\alpha(\|x\|), \\
\lambda^{-\mu} V\left(\Lambda_{r}(\lambda) x\right)=V(x), \mu>r_{\max },
\end{gathered}
$$

$\forall x \in \mathbb{R}^{n}$ and $\forall \lambda>0$, for some $\alpha_{1}, \alpha_{2} \in \mathcal{K}_{\infty}$ and $\alpha \in \mathcal{K}$;

- there is a compact strictly positively invariant set $S$ containing the origin (i.e. $X\left(t, x_{0}\right) \in S \backslash \partial S$ for all $t>0$ and all $x_{0} \in \partial S$ ).

The requirement on continuity of the function $f$ has been relaxed in [25] (the function $V$ can still be selected smooth).

Theorem 2 ([26]): If (1) is $\mathbf{r}$-homogeneous of degree $\nu$ and asymptotically stable at the origin, then it is

(i) globally finite-time stable at the origin if $\nu<0$;

(ii) globally exponentially stable at the origin if $\nu=0$;

(iii) globally fixed-time stable with respect to the unit ball $B_{r}(1)$, if $\nu>0$.

\section{FINITE-TIME OBSERVER DESIGN}

In order to introduce later a distributed finite-time observer, let us briefly recall a centralized concept of finite-time estimator from [9].

For this purpose consider a chain of integrators:

$$
\dot{x}=A x, \quad y=c^{\mathrm{T}} x,
$$

where $x \in \mathbb{R}^{n}$ is the state, $y \in \mathbb{R}$ is the output of the system and the matrix $A \in \mathbb{R}^{n \times n}$ and the output vector $c \in \mathbb{R}^{n}$ are given by

$$
A=\left[\begin{array}{ccccc}
0 & 1 & 0 & \ldots & 0 \\
0 & 0 & 1 & \ldots & 0 \\
& & \vdots & & \\
0 & 0 & \ldots & 0 & 1 \\
0 & 0 & \ldots & 0 & 0
\end{array}\right], \quad c=\left[\begin{array}{c}
1 \\
0 \\
\vdots \\
0
\end{array}\right] .
$$

Note that any fully observable system can be transformed into a chain of integrators modulo an output injection, as well as some nonlinear systems [27].

Based on Luenberger observer design, a finite-time observer has been constructed in [9] as

$$
\dot{z}=A z+\operatorname{diag}(\ell)\left\lceil\left(1_{n}\left(c^{\mathrm{T}} z-y\right)\right\rfloor^{\gamma},\right.
$$

where $z \in \mathbb{R}^{n}$ is the estimate of the state $x$, the observer gain $\ell \in \mathbb{R}^{n}$ and $\gamma \in \mathbb{R}^{n}$ are defined later. Introducing the observer error as

$$
e=z-x
$$


yields with (2) and (3) the error system:

$$
\dot{e}=f(e)=A e+\operatorname{diag}(\ell)\left[1_{n} c^{\mathrm{T}} e\right\rfloor^{\gamma} .
$$

Next, let us briefly sketch the proof of finite-time stability of the system in (4). These arguments will be reused later in the distributed scenario.

\section{A. Homogeneity}

First, we check that the error system (4) is homogeneous, meaning that $f(e)$ verifies the restrictions imposed in Definition 2 for some homogeneity degree $\nu<0$ and weights $\mathbf{r}$. For this goal, choose

$$
r_{s}=1+(s-1) \nu, s=\overline{1, n},
$$

then $r_{s}>0$ for $-\frac{1}{n}<\nu<0$. For each $s=\overline{1, n-1}$ this gives

$$
f_{s}\left(\Lambda_{r}(\lambda) e\right)=\lambda^{r_{s+1}} e_{s+1}+\ell_{s} \lambda^{r_{1} \gamma_{s}}\left\lceil e_{1}\right\rfloor^{\gamma_{s}}
$$

and

$$
f_{n}\left(\Lambda_{r}(\lambda) e\right)=\ell_{n} \lambda^{r_{1} \gamma_{n}}\left\lceil e_{1}\right\rfloor^{\gamma_{n}} .
$$

Therefore, for $\gamma=\left[\begin{array}{lll}\gamma_{1} & \ldots & \gamma_{n}\end{array}\right]^{\mathrm{T}}$ in (3) with

$$
\gamma_{s}=\frac{r_{s+1}}{r_{1}}=1+s \nu, \quad s=\overline{1, n-1} \text { and } \gamma_{n}=1+n \nu
$$

the error system (4) is homogeneous with a negative degree $\nu$. In addition, $0<\gamma_{s}<1$.

\section{B. Stability}

Next, we show that (4) has a strictly positively invariant set. Rewrite the system (4) as follows:

$$
\dot{e}=\left(A+\ell c^{\mathrm{T}}\right) e+\operatorname{diag}(\ell)\left(\left\lceil 1_{n} e_{1}\right\rfloor^{\gamma}-1_{n} e_{1}\right) .
$$

Let us analyze the Lyapunov function

$$
V(e)=e^{\mathrm{T}} P e,
$$

with $P=P^{\mathrm{T}}>0$ as the solution of the Lyapunov equation

$$
\tilde{A}^{\mathrm{T}} P+P \tilde{A}+Q=0,
$$

with $\tilde{A}=A+\ell c^{\mathrm{T}}$, a matrix $Q=Q^{\mathrm{T}}>0$ and under an auxiliary not restricting condition

$$
P \geq I_{n} .
$$

Since the system is observable, then always there exist such $\ell, P$ and $Q$ that these linear matrix inequalities are satisfied. The time derivative of $V$ for (6) can be written as follows:

$$
\begin{aligned}
\dot{V}(e) & =-e^{\mathrm{T}} Q e+2 e^{\mathrm{T}} P \operatorname{diag}(\ell)\left(\left\lceil 1_{n} e_{1}\right\rfloor^{\gamma}-1_{n} e_{1}\right) \\
& \leq-\frac{\lambda_{\min }(Q)}{\lambda_{\max }(P)} V+2\|e\|\|P \operatorname{diag}(\ell)\|\left\|\left\lceil 1_{n} e_{1}\right\rfloor^{\gamma}-1_{n} e_{1}\right\|
\end{aligned}
$$

Considering $e \in S=\left\{e \in \mathbb{R}^{n}: V(e)=1\right\}$ (i.e. $\|e\|^{2} \leq$ $\lambda_{\min }^{-1}(P) \leq 1$ ) we obtain

$$
\begin{aligned}
\dot{V} & \leq-\frac{\lambda_{\min }(Q)}{\lambda_{\max }(P)}+2\|P \operatorname{diag}(\ell)\| \sqrt{\sum_{s=1}^{n}\left(\left\lceil e_{1}\right\rfloor \gamma_{s}-e_{1}\right)^{2}} \\
& =-\frac{\lambda_{\min }(Q)}{\lambda_{\max }(P)}+2\|P \operatorname{diag}(\ell)\| \sqrt{\sum_{s=1}^{n}\left(\left|e_{1}\right|^{\gamma_{s}}-\left|e_{1}\right|\right)^{2}} .
\end{aligned}
$$

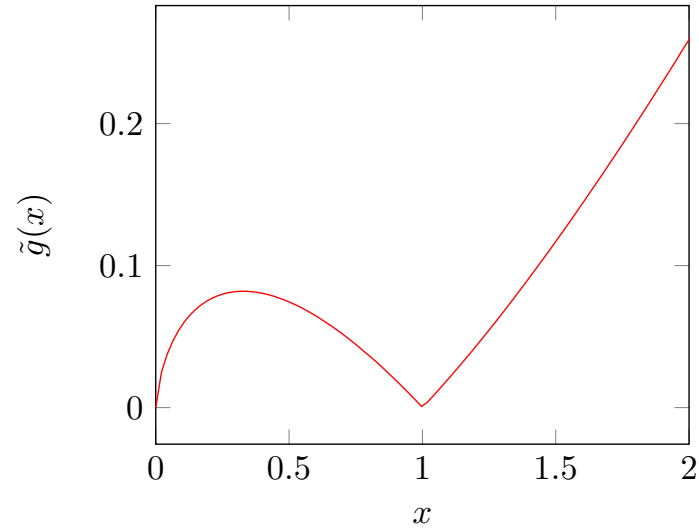

Fig. 1. Example of $\tilde{g}(x)$ with $\alpha=0.8$

Note that for $\nu=0$ the above expression is reduced to

$$
\dot{V} \leq-\frac{\lambda_{\min }(Q)}{\lambda_{\max }(P)} .
$$

Then by continuity it is also satisfied for a negative $\nu$ close to zero since $\frac{\lambda_{\min }(Q)}{\lambda_{\max }(P)}>0$.

To derive an expression for $\nu$, which guarantees that (7) is negative definite on $S$, we have to calculate an upper bound for $\left|e_{1}\right|^{\gamma_{s}}-\left|e_{1}\right|$ taking in mind that $\left|e_{1}\right| \in[0,1]$ for $e \in S$. To get an idea, consider the function $\tilde{g}(x)=\left|x^{\alpha}-x\right|$ for $x=[0,1]$. An example of $\tilde{g}$ is given in Fig. 1 for $\alpha=0.8$. Inspired by the graph of $\tilde{g}$, we apply the Mean Value Theorem:

$$
g(a)-g(b)=g^{\prime}(\theta)(a-b), \quad \theta \in[a, b]
$$

to the function $g: \alpha \mapsto x^{\alpha}$, considering $x \in[0,1]$ as a parameter and $\alpha \in[0,1)$ as the argument. For $\alpha \in[0,1)$, we obtain

$$
g(\alpha)-g(1)=\xi(x, \theta)(\alpha-1)
$$

with $\xi(x, \theta)=\ln (x) x^{\theta}$ for some $\theta \in[\alpha, 1]$. For any such fixed $\theta, \xi(0, \theta)=\xi(1, \theta)=0$ and $\xi(x, \theta) \leq 0$ for any $x \in[0,1]$. It is easy to check that the minimal value of $\xi$ is reached at

$$
x_{\mathrm{opt}}=\exp \left(-\theta^{-1}\right), \xi\left(x_{\mathrm{opt}}, \theta\right)=-\theta^{-1} \exp (-1) .
$$

Thus we can use the bound

$$
\left|e_{1}\right|^{\gamma_{s}}-\left|e_{1}\right| \leq \exp (-1) \frac{1-\gamma_{s}}{\gamma_{s}}=\exp (-1) \frac{-s \nu}{1+s \nu} .
$$

Additionally, using

$$
\left|\frac{s \nu}{1+s \nu}\right| \leq\left|\frac{n \nu}{1+n \nu}\right|
$$

for $s=\overline{1, n}$ leads to

$$
\begin{aligned}
\dot{V} & \leq-\frac{\lambda_{\min }(Q)}{\lambda_{\max }(P)}+2 \exp (-1)\|P \operatorname{diag}(\ell)\| \sqrt{\sum_{s=1}^{n}\left(\frac{n \nu}{1+n \nu}\right)^{2}} \\
& =-\frac{\lambda_{\min }(Q)}{\lambda_{\max }(P)}+2 \exp (-1)\|P \operatorname{diag}(\ell)\| \sqrt{n} \frac{n|\nu|}{1+n \nu} .
\end{aligned}
$$




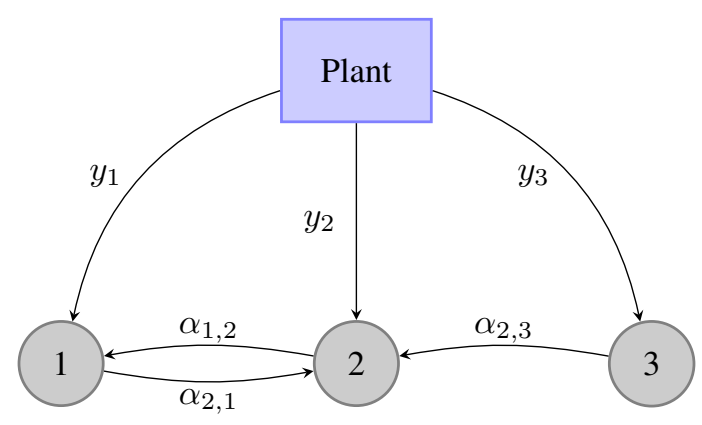

Fig. 2. Scheme depicting a distributed observer with $N=3$

Now a bound for $\nu$ can be selected as

$$
|\nu|<\frac{\eta}{n(\sqrt{n}+\eta)}, \quad \eta=\frac{\exp (1)}{2} \frac{\lambda_{\min }(Q)}{\lambda_{\max }(P)\|P \operatorname{diag}(\ell)\|},
$$

which ensures that $\dot{V}(e)<0$ for $e \in S$ and that the set $S$ is strictly positively invariant for (4). Thus by Theorem 1 the system is globally asymptotically stable, and since $\nu<0$, it is globally finite-time stable by Theorem 2 .

\section{Distributed OBSERVER}

Now we turn to the networked observer case. In this setting, the plant has $N>1$ outputs available for measurements:

$$
\dot{x}=A x, \quad y_{i}=c_{i}^{\mathrm{T}} x, \quad i=\overline{1, N},
$$

each having a different output matrix $c_{i} \in \mathbb{R}^{n}, C^{\mathrm{T}}=$ $\left[c_{1} \ldots c_{N}\right]$. The distributed $N$ observers in this work are selected in the following form for each $i=\overline{1, N}$ :

$\dot{z}_{i}=A z_{i}+\operatorname{diag}\left(\ell_{i}\right)\left[1_{n}\left(c_{i}^{\mathrm{T}} z_{i}-y_{i}\right)\right]^{\gamma_{i}}+\sum_{j \in \mathcal{N}_{i}} \alpha_{i, j}\left[z_{j}-z_{i}\right\rfloor^{\beta}$,

where $z_{i} \in \mathbb{R}^{n}$ is an estimate of the state generated by the $i^{\text {th }}$ observer; $\ell_{i} \in \mathbb{R}^{n}, \alpha_{i, j} \in \mathbb{R}_{+}, \gamma_{i} \in \mathbb{R}^{n}$ and $\beta \in \mathbb{R}^{n}$ are observer parameters selected later; the observers are connected in a graph and $\mathcal{N}_{i}$ denotes the set of neighboring nodes, i.e. $j \in \mathcal{N}_{i}$ if there is a directed edge from $j$ to $i\left(\alpha_{i, j}=0\right.$ otherwise), see an example in Fig. 2 with $\mathcal{N}_{1}=\{2\}, \mathcal{N}_{2}=\{1,3\}$ and $\mathcal{N}_{3}=\emptyset$. Of course, if there exists one $c_{i}$ such that the system (8) is observable from that output, then a distributed structure becomes redundant, but if the pair $(A, C)$ is observable and each separate pair $\left(A, c_{i}^{\mathrm{T}}\right)$ is not, then coupling $\alpha_{i, j} \neq 0$ between the observers is obligatory.

For each node, the observer error is

$$
e_{i}=z_{i}-x
$$

and the error system is

$$
\dot{e}_{i}=A e_{i}+\operatorname{diag}\left(\ell_{i}\right)\left\lceil 1_{n} c_{i}^{\mathrm{T}} e_{i}\right\rfloor^{\gamma_{i}}+\sum_{j \in \mathcal{N}_{i}} \alpha_{i, j}\left\lceil\left(e_{j}-e_{i}\right)\right\rfloor^{\beta} .
$$

The goal of this section is to propose conditions under which the interconnected systems in (10) are finite-time stable. The construction below will follow the main ideas given in
Section III for the design and the analysis of a finite-time centralized observer (3).

Similar to the SISO case we will assume that $A$ and $c_{i}$ are in a nominal form:

$$
\begin{aligned}
& A=\left[\begin{array}{ccccc}
0 & \delta_{1} & 0 & \ldots & 0 \\
0 & 0 & \delta_{2} & \ldots & 0 \\
& & \vdots & & \\
0 & 0 & \ldots & 0 & \delta_{n-1} \\
0 & 0 & \ldots & 0 & 0
\end{array}\right], \quad \delta_{i} \in\{0,1\},
\end{aligned}
$$

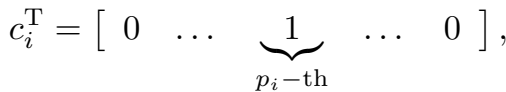

where $p_{i} \in[1, n]$ for any $i=\overline{1, N}$. At least one $\delta_{i}=0$ makes it necessary to introduce cross-coupling terms in (9) (in other words, each observer of the form (3) is not able to estimate the full-state, but using all outputs, the system can be observable).

\section{A. Homogeneity}

Once again, we look for the conditions of homogeneity. For $s=\overline{1, n-1}$, this means that

$$
\begin{aligned}
\lambda^{\nu} \lambda^{1+(s-1) \nu} \dot{e}_{i, s} & =\lambda^{1+s \nu} \delta_{s} e_{i, s+1}-\ell_{i, s} \lambda^{\left(1+\left(p_{i}-1\right) \nu\right) \gamma_{i, s}}\left[e_{p_{i}, s}\right]^{\gamma_{i, s}} \\
& +\sum_{j \in \mathcal{N}_{i}} \alpha_{i, j} \lambda^{(1+(s-1) \nu) \beta_{s}}\left\lceil\left(e_{j, s}-e_{i, s}\right)\right]^{\beta_{s}}
\end{aligned}
$$

has to be fulfilled, leading to the following result:

Lemma 3: The system (10) with (11), (12) is homogeneous with respect to the weights in (5) for

$$
\gamma_{i, s}=\frac{1+s \nu}{1+\left(p_{i}-1\right) \nu}, \quad \beta_{s}=\frac{1+s \nu}{1+(s-1) \nu},
$$

where $i=\overline{1, N}$ and $s=\overline{1, n}$.

Note that the properties $0<\beta_{s}<1$ and $\gamma_{i, s}>0$ are satisfied by construction.

\section{$B$. The main result formulation}

For $\nu=0$, the observers in (9) are standard linear Luenberger observers with couplings and the asymptotic stability of the network can be evaluated using the error system

$$
\dot{E}=W E
$$

for the large scale error $E^{\mathrm{T}}=\left[\begin{array}{lll}e_{1}^{\mathrm{T}} & \ldots & e_{N}^{\mathrm{T}}\end{array}\right] \in \mathbb{R}^{N n}$ with

$$
\begin{aligned}
& W=\operatorname{diag}\left\{A+\ell_{i} c_{i}^{\mathrm{T}}\right\}_{i=1}^{N}+H \otimes I_{n}, \\
& H=\left[h_{i j}\right]_{i, j=1}^{N}, \quad h_{i j}= \begin{cases}-\sum_{k \in \mathcal{N}_{i}} \alpha_{i, k} & \text { for } i=j, \\
\alpha_{i, j} & \text { for } i \neq j .\end{cases}
\end{aligned}
$$

We now assume that with $\gamma_{i}=\beta=1_{n}$ and appropriate gains $l_{i}, \alpha_{i, j}$ in (9) an asymptotically converging observer can be designed (possible using methods referenced in the introduction). This justifies following assumption:

Assumption 1: There exist solutions $P=P^{\mathrm{T}}>0$ and $Q=Q^{\mathrm{T}}>0$ of the following system of linear matrix inequalities:

$$
\begin{gathered}
W^{\mathrm{T}} P+P W+Q=0, \\
P \geq I_{N n} .
\end{gathered}
$$


Now we are in position to formulate the main result of this paper:

Theorem 4: Let Assumption 1 be satisfied, then the estimation error of the observer network (9) with (8), (11), (12) is globally finite time stable, provided that $\gamma_{i}, \beta$ are chosen according to Lemma 3 and

$|\nu| \leq \frac{(2 n-1) h-(a+b)+\sqrt{h^{2}-2(a-b) h+(a+b)^{2}}}{2[(a-n h)(n-1)+b n]}$,

where

$$
\begin{aligned}
a & =\left\|P \operatorname{diag}\left\{\operatorname{diag}\left(\ell_{i}\right)\right\}_{i=1}^{N}\right\| \exp (-1) \sqrt{N n} \\
b & =\|P M\| \ln (2) 2 N \sqrt{n} \\
h & =\frac{\lambda_{\min }(Q)}{2 \lambda_{\max }(P)}, \\
M & =\operatorname{diag}\left\{\alpha_{i}^{\mathrm{T}} \otimes I_{n}\right\}_{i=1}^{N}, \quad \alpha_{i}^{\mathrm{T}}=\left[\begin{array}{lll}
\alpha_{i 1} & \ldots & \alpha_{i N}
\end{array}\right]
\end{aligned}
$$

and $P, Q$ are the solutions of (15).

Remark 5: The proof follows similar lines as in the SISO case, the difference being the range of values when applying the Mean-Value Theorem. The assessment (16) is obtained by replacing the variables $s$ and $p_{i}$ by their limit values 1 and $n$ which is a rather conservative substitution allowing an upper bound (16) to be derived explicitly.

Remark 6: In this work, only the noise-free case is studied for the observer design. Nevertheless, following [28]-[31], since under (13) the error system (10) is homogeneous of negative degree and globally finite-time stable, then the distributed observer (9) is robust with respect to additive disturbances in the right-hand side of (8), measurement noises and delays in the output channel.

Remark 7: A fixed-time distributed observer might be designed by introducing additional terms of positive homogeneity degree in (9):

$$
\begin{aligned}
\dot{z}_{i}=A z_{i}+\operatorname{diag}\left(\ell_{i}\right) \sum_{k=1}^{2}\left\lceil 1_{n}\left(c_{i}^{\mathrm{T}} z_{i}-y_{i}\right)\right\rfloor^{\gamma_{i}^{k}} & \\
& \quad+\sum_{j \in \mathcal{N}_{i}} \alpha_{i, j} \sum_{k=1}^{2}\left\lceil z_{j}-z_{i}\right\rfloor^{\beta^{k}},
\end{aligned}
$$

where, following (13):

$$
\gamma_{i, s}^{k}=\frac{1+s \nu_{k}}{1+\left(p_{i}-1\right) \nu_{k}}, \quad \beta_{s}^{k}=\frac{1+s \nu_{k}}{1+(s-1) \nu_{k}}
$$

for $i=\overline{1, N}, s=\overline{1, n}$ with $-\frac{1}{n}<\nu_{1}<0$ and $\nu_{2}>0$. The proof of fixed-time stability of this observer would follow local homogeneity arguments (as in [7], [32]) and by repeating the main steps used in the proof of Theorem 4 .

\section{EXAMPLE}

Let us consider the simple example with $n=4$ and $N=2$ :

$$
\dot{x}=\left[\begin{array}{llll}
0 & 1 & 0 & 0 \\
0 & 0 & 0 & 0 \\
0 & 0 & 0 & 1 \\
0 & 0 & 0 & 0
\end{array}\right] x, c_{1}=\left[\begin{array}{l}
1 \\
0 \\
0 \\
0
\end{array}\right], c_{2}=\left[\begin{array}{l}
0 \\
0 \\
1 \\
0
\end{array}\right],
$$

consequently $\delta_{2}=0$ in (11) for this case.

The observers are fully connected in a graph with $\alpha_{1,2}=$ $\alpha_{2,1}=1.7$. The observer gains are chosen as

$$
\begin{aligned}
& \ell_{1}=\left[\begin{array}{llll}
-3.7 & -3.7 & 0 & 0
\end{array}\right]^{\mathrm{T}}, \\
& \ell_{2}=\left[\begin{array}{llll}
0 & 0 & -3.7 & -3.7
\end{array}\right]^{\mathrm{T}} .
\end{aligned}
$$

Solving (15) with $Q=I_{n N}$ leads to a positive definite solution. Next, using (16) we calculate $\nu=-0.0046$.

As already stated in Remark 5, the resulting $\nu$ is rather conservative and as a result the corresponding settling time is too large to show the finite-time behavior visually. If compared to the linear observer (i.e. $\nu=0$ ) the error will reach asymptotically machine precision only slightly later. However, following Remark 5 a more accurate assessment of $\nu$ is possible. For instance, in Fig. 3 the error norm $\|E(t)\|$ is plotted in a logarithmic scale with $\nu=-0.1$ and compared to the linear observer with the same gains, clearly showing the finite time behavior for the former and the exponential decrease for the latter.

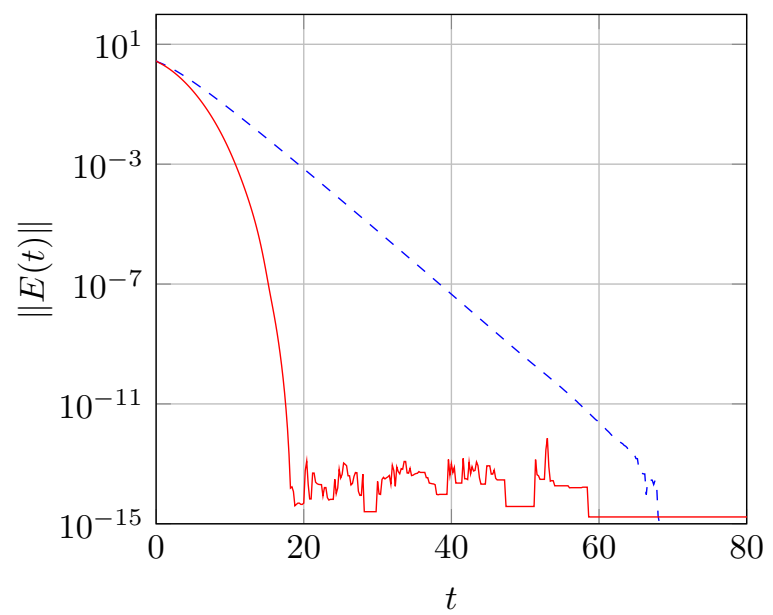

Fig. 3. Comparison of the error for the linear (dashed) and finite-time (solid) observer.

\section{CONCLUSION}

The problem of distributed observer design for a linear system was studied, and two observer structures are proposed, converging in a finite or in a fixed time. Due to homogeneity, the designed observers possess input-to-state stability properties with respect to additive perturbations and measurement noises in the plant equations. In the finite-time case the estimator has also bounded trajectories for any delay in the output channel. The detailed substantiations of these properties are left for future works, as well as the calculation of estimates on the degrees of homogeneity for the fixed-time observer found in the present paper.

\section{REFERENCES}

[1] J. L. Crassidis and J. L. Junkins, Optimal Estimation of Dynamic Systems, 2nd. CRC Press, 2012.

[2] D. G. Luenberger, Introduction to dynamic systems: theory, models, and applications. Wiley, 1979. 
[3] H. K. Khalil, Nonlinear Systems, ser. NJ 07458. Upper Saddle River: Prentice-Hall, 1996.

[4] W. Perruquetti and J. P. Barbot, Sliding Mode Control in Engineering. Marcel Dekker Hardcover, 2002.

[5] V. Andrieu, L. Praly, and A. Astolfi, "High gain observers with updated gain and homogeneous correction terms," Automatica, vol. 45, no. 2, pp. 422-428, 2009.

[6] J. H. Ahrens and H. K. Khalil, "High-gain observers in the presence of measurement noise: A switched-gain approach," Automatica, vol. 45, no. 4, pp. 936-943, 2009.

[7] V. Andrieu, L. Praly, and A. Astolfi, "Homogeneous Approximation, Recursive Observer Design, and Output Feedback," SIAM J. Control Optimization, vol. 47, no. 4, pp. 1814-1850, 2008.

[8] A. Levant, "Homogeneity approach to high-order sliding mode design," Automatica, vol. 41, no. 5, pp. 823-830, 2005.

[9] W. Perruquetti, T. Floquet, and E. Moulay, "Finite-time observers: Application to secure communication," IEEE Transactions on Automatic Control, vol. 53, no. 1, pp. 356-360, Feb. 2008.

[10] H. Ríos and A. Teel, "A hybrid observer for uniform finite-time state estimation of linear systems," in In Proc. 55th IEEE Conference on Decision and Control (CDC), Las Vegas, 2016.

[11] F. Lopez-Ramirez, D. Efimov, A. Polyakov, and W. Perruquetti, "Fixed-time output stabilization of a chain of integrators," 2016 IEEE 55th Conference on Decision and Control (CDC), Dec. 2016.

[12] F. Pasqualetti, F. Dörfler, and F. Bullo, "Attack detection and identification in cyber-physical systems," IEEE Transactions on Automatic Control, vol. 58, no. 11, pp. 2715-2729, Nov. 2013.

[13] V. Ugrinovskii, "Conditions for detectability in distributed consensus-based observer networks," IEEE Transactions on Automatic Control, vol. 58, no. 10, pp. 2659-2664, Oct. 2013.

[14] R. Olfati-Saber, "Distributed kalman filtering and sensor fusion in sensor networks," in Networked Embedded Sensing and Control: Workshop NESC'05: University of Notre Dame, USA October 2005 Proceedings, P. J. Antsaklis and P. Tabuada, Eds. Berlin, Heidelberg: Springer Berlin Heidelberg, 2006, pp. 157-167.

[15] M. Deghat, V. Ugrinovskii, I. Shames, and C. Langbort, "Detection of biasing attacks on distributed estimation networks," in 2016 IEEE 55th Conference on Decision and Control (CDC), Dec. 2016, pp. 2134-2139.

[16] A. B. Alexandru, S. Pequito, A. Jadbabaie, and G. J. Pappas, "Decentralized observability with limited communication between sensors," in 2016 IEEE 55th Conference on Decision and Control (CDC), Las Vegas, Dec. 2016, pp. 885-890.

[17] T. Kim, H. Shim, and D. D. Cho, "Distributed luenberger observer design," in 2016 IEEE 55th Conference on Decision and Control (CDC), Las Vegas, Dec. 2016, pp. 6928-6933.

[18] S. P. Bhat and D. S. Bernstein, "Geometric homogeneity with applications to finite-time stability," Mathematics of Control, Signals and Systems, vol. 17, pp. 101-127, 2005.

[19] E. Roxin, "On finite stability in control systems," Rendiconti del Circolo Matematico di Palermo, vol. 15, pp. 273-283, 1966.

[20] A. Polyakov, "Nonlinear feedback design for fixed-time stabilization of linear control systems," IEEE Transactions on Automatic Control, vol. 57, no. 8, pp. 2106-2110, 2012.

[21] V. Zubov, "On systems of ordinary differential equations with generalized homogenous right-hand sides," Izvestia vuzov. Mathematica., vol. 1, pp. 80-88, 1958 (in Russian).

[22] A. Bacciotti and L. Rosier, Lyapunov Functions and Stability in Control Theory, 2nd. Springer, Berlin, 2005.

23] L. Rosier, "Homogeneous Lyapunov function for homogeneous continuous vector field," Systems \& Control Letters, vol. 19, pp. 467473, 1992.

[24] S. P. Bhat and D. S. Bernstein, "Finite time stability of continuous autonomous systems," SIAM J. Control Optim., vol. 38, no. 3, pp. 751-766, 2000.

[25] E. Bernuau, A. Polyakov, D. Efimov, and W. Perruquetti, "On extension of homogeneity notion for differential inclusions," in Proceeding of the European Control Conference, Zurich, 2013, pp. 2204-2209.

[26] E. Bernuau, D. Efimov, W. Perruquetti, and A. Polyakov, "On homogeneity and its application in sliding mode control," Journal of the Franklin Institute, vol. 351, no. 4, pp. 1866-1901, 2014.

[27] A. Isidori and A. Krener, "On feedback equivalence of nonlinear systems," Systems \& Control Letters, vol. 2, no. 2, pp. 118-121, 1982.
[28] E. Bernuau, A. Polyakov, D. Efimov, and W. Perruquetti, "Verification of ISS, iISS and IOSS properties applying weighted homogeneity," Systems \& Control Letters, vol. 62, pp. 1159-1167, 2013.

[29] D. Efimov, W. Perruquetti, and J.-P. Richard, "Development of homogeneity concept for time-delay systems," SIAM Journal on Control and Optimization, vol. 52, no. 3, pp. 1547-1566, 2014

[30] D. Efimov, A. Polyakov, W. Perruquetti, and J. P. Richard, "Weighted homogeneity for time-delay systems: Finite-time and independent of delay stability," IEEE Transactions on Automatic Control, vol. 61, no. 1, pp. 210-215, Jan. 2016.

[31] K. Zimenko, D. Efimov, A. Polyakov, and W. Perruquetti, "A note on delay robustness for homogeneous systems with negative degree," Automatica, vol. 79, pp. 178-184, 2017.

[32] D. Efimov and W. Perruquetti, "On conditions of oscillations and multi-homogeneity," Mathematics of Control, Signals, and Systems, vol. 28 , no. 1 , pp. $1-37,2015$ 